\title{
Dielectric properties of bionano-composites modified by fish scales
}

\author{
Gojayev EM 1,* and Salimova VV 2 \\ ${ }^{1}$ Head of Department of Physics engineering and Electronica, Azerbaijan Technical University, Baku, Azerbaijan. \\ 2 The department of Physics and its education, Sumgait State University, Azerbaijan.
}

Global Journal of Engineering and Technology Advances, 2021, 09(02), 043-046

Publication history: Received on 30 June 2021; revised on 22 August 2021; accepted on 24 August 2021

Article DOI: https://doi.org/10.30574/gjeta.2021.9.2.0113

\begin{abstract}
The presented work presents the results of studying the temperature dependences of the dielectric constant and the tangent of the dielectric loss angle of biocomposites modified with fish scales. It was found that with an increase in the volumetric content of the bio-filler, the dielectric constant of the biocomposites increases, and the tangent of the dielectric loss angle decreases. By varying the volumetric content of the bio-filler, it is possible to control the properties of biocomposites in the desired direction.
\end{abstract}

Keywords: Dielectric constant; Tangent of dielectric loss angle; Biocomposites; Bio-filler; Fish scales

\section{Introduction}

The existing technological practice of manufacturing composite materials indicates the possibility of obtaining dielectric materials with various properties based on a combination of different types of polymers. Distinguish between thermodynamically and technologically compatible polymers. Thermodynamically compatible polymers with additives completely dissolve in each other, but there are extremely few such pairs of polymeric substances [1-3]. There are many modifications of the obtained thermodynamically incompatible polymers, the physicochemical and electrophysical properties of which satisfy a set of requirements for the finished product and guarantee its long-term operation. Such polymers are called technologically compatible. In this case, the possibility of obtaining a product with specified properties largely depends on the choice of polymer and filler components and on the rational conduct of technological processes. The introduction of a filler into polymers is accompanied by the appearance of new artificial nuclei of crystallization and centers of trapping of an electric charge carrier, which can contribute to an increase in the surface charge density and their lifetime. In this work, fish scales were used as modifying additives.

\section{Experimental part}

To obtain biocomposites, we used M-158 high-pressure polyethylene as a matrix, and fish scales as a bio-filler. The fish scales powder was obtained as follows: at the beginning, fish scales (FS) were thoroughly cleaned and dried, gradually increasing the temperature from room temperature to $330 \mathrm{~K}$ with exposure at this temperature for 10 minutes. The scales of the fish dried in this way were ground into powder in a special mill. The content of the bio-filler in the composites varied from 1 to $15 \mathrm{vol}$.\%. Biocomposites were prepared from a homogeneous mixture of powders of the matrix and filler components using a heated press at a temperature of $420 \mathrm{~K}$ and a pressure of $10 \mathrm{MPa}$ [4]. The quenching crystallization mode occurs faster when the samples are cooled in a water-ice mixture.

At the same temperature, due to the compaction of a homogeneous mixture, the pressure slowly increased to $15 \mathrm{MPa}$. Sample sizes: thickness about $170 \mu \mathrm{m}$, diameter of the obtained samples $50 \mathrm{~mm}$. A schematic diagram of a device for

\footnotetext{
${ }^{*}$ Corresponding author: Gojayev EM

Head of Department of Physics engineering and Electronica, Azerbaijan Technical University, Baku, Azerbaijan. 
measuring the tangent of the dielectric loss angle $(\operatorname{tg} \delta)$ and electrical capacitance $(\mathrm{C})$ of the samples under study is shown in Figure 1. Sample 1 is placed in an insulating chamber (8), between measuring electrodes 2 and 3 . Polished brass diskswere used as measuring electrodes. The diameter of the upper electrode was $25 \mathrm{~mm}$ and the diameter of the lower electrode was $50 \mathrm{~mm}$. The lower electrode 3 must be grounded with 9 . The upper electrode 2 is pressed against the sample with a load 5 using screws 4 . The sample was heated using an oven 7 powered by a trilator system 12 . The sample temperature was measured using an electronic thermometer 10 and a copper-coupled thermocouple 6 . The tangent of the dielectric loss angle and the electrical capacitance of the samples under study were measured using a P589 variable bridge 11 in the temperature range of $290-390 \mathrm{~K}$ at $1 \mathrm{kHz}$. The bridge was powered from a $220 \mathrm{~V}$ and frequency $50 \mathrm{~Hz}$.Using the experimental data of the electrical capacity and knowing the geometric dimensions of the sample, the dielectric constant is determined using the formula. The electrical contact of the electrodes was provided by smearing silver paste.The electrical capacity of the sample was determined with the formula

$$
C=\varepsilon \varepsilon_{0} \frac{S}{d} \Rightarrow \varepsilon=\frac{C d}{\varepsilon_{0} S} .
$$

where, $C$ is the electrical capacity of the sample, $\varepsilon_{0}=8.85 \cdot 10^{-12} \mathrm{~F} / \mathrm{m}$ is the electrical constant, $d$ is the thickness of the sample, $S, \mathrm{~m}^{2}$ is the surface area of the sample [5].

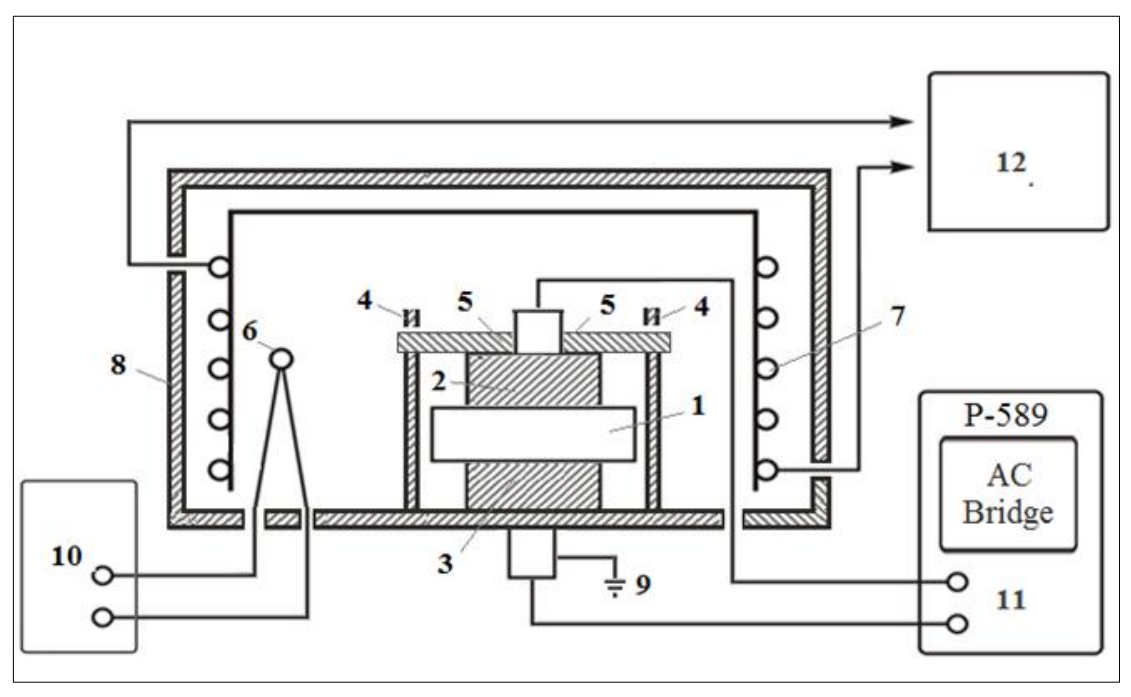

Figure 11 - sample, 2 - upper electrode, 3 - lower electrode, 4- screw, 5 - load, 6- thermocouple, 7 - furnace, 8 isolation chamber, 9 - grounding, 10-electronic thermometer, 12-trilator system, 11- AC bridge P-58

\section{Results and discussion}

It is known that the dielectric properties of polymer insulation are closely related to the structure of the polymer. Naturally, one should expect a change in the dielectric constant and the tangent of the dielectric loss angle of the highpressure polyethylene film with various additives. To clarify the possibility of using a polymer as a dielectric and to obtain information about the thermal motion and molecular structure of the polymer, it is necessary to know not only the value of dielectric losses, but also the nature and quantitative characteristics depending on temperature, composition, amount of fillers, irradiation, and other factors.

In this work, we have investigated the temperature dependences of the dielectric constant and the tangent of the dielectric loss angle of bionanocomposites according to the general formula LDPE + x vol\% FS. The results of studying the dielectric parameters are shown in Fig. 2. As can be seen from Fig.2a, the tangent of the dielectric loss angle decreases with an increase in the volumetric content of the bio-filler. The tangent of the dielectric loss angle at the maximum, depending on the content of fish scales, decreases. The $\operatorname{tg}(\mathrm{T})$ curves in the LDPE+FS dependences show one clearly pronounced maximum in the temperature range of 360-370 K.

There are several types of dielectric losses in polymers, of which, first of all, dipole-segmental, dipole-elastic, dipolegroup and dipole-radical can be distinguished. All of the above losses are mainly related to amorphous polymers. In crystalline polymers, it is more difficult to attribute dielectric losses to one form or another. For amorphous polymers, 
areas of maximum $\mathrm{tg}$ are possible associated with melting of polymers, movement of various groups in crystalline regions, etc.

Polyethylene belongs to non-polar polymers, therefore, $\mathrm{tg}$ is very small and weakly depends on frequency and temperature, practically does not have dipole polarization. Crystallizing nonpolar polymers exhibit relatively weak $\mathrm{tg}$ maxima associated with the presence of impurity polar groups. As can be seen from Fig. 2a, for the studied biocomposites, $t g$ begins at high temperatures, but these changes are insignificant in relation to the LDPE samples without additive. It is likely that the additives introduced into the LDPE composition affect the crystallization kinetics and significantly increase the packing density of macromolecules. As a result, molecular interactions in the polymer chains increase and the movement of the kinematic segments is impeded, that is, some of the impurity polar radicals lose their mobility, falling into the crystalline regions.
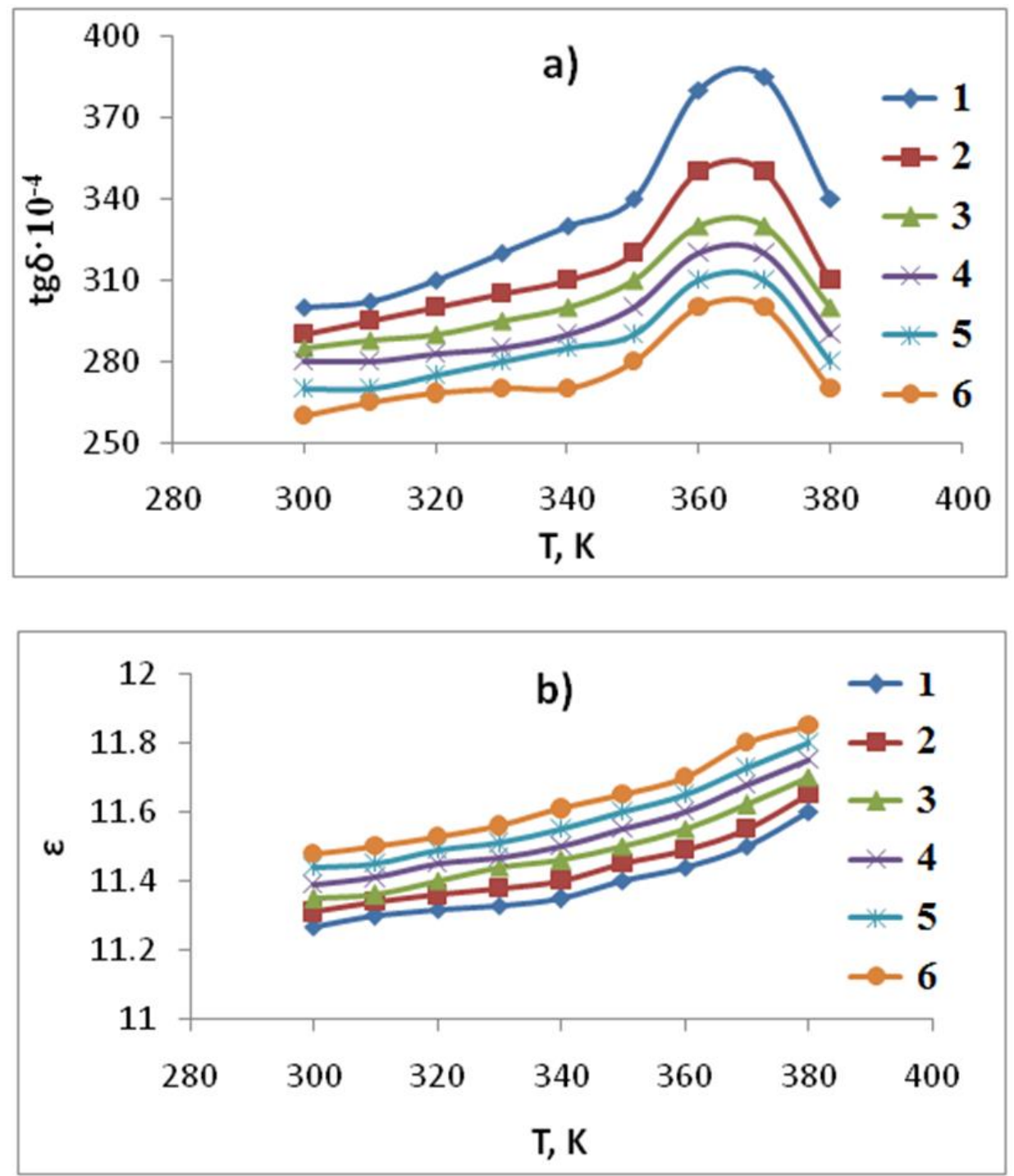

Figure 2 Temperature dependence of the tangent of the dielectric loss angle (a) and the dielectric constant (b) of the biocomposites LDPE + x vol\%FS

Where $1-x=1,2-x=3,3-x=5,4-x=7,5-x=10,6-x=15$.

The results of studying the temperature dependence of the dielectric constant of LDPE + FS biocomposites are shown in Fig. 2b. As follows from the figure, with an increase in temperature from 300 to $380 \mathrm{~K}$, the dielectric constant of the studied biocomposites with fillers 1,3,5,7,10 and 15\% of the volumetric content of fish scales increases monotonically. Weak maxima are observed only at a temperature of $320 \mathrm{~K}$. An increase in the dielectric constant of the studied biocomposites is observed with an increase in the volumetric content of the bio-filler. 


\section{Conclusion}

Analyzing the obtained experimental data, we can conclude that the proposed modifiers of biological origin contribute to the modification of dielectric properties and improvement of their stability. Note that when a physical structurant is introduced into the polymer, which does not lead to the formation of additional polar groups and impurities, and also promotes the formation of a homogeneous structure, the dielectric loss $\operatorname{tg} \delta(\mathrm{T})$ mainly decreases and the relaxation time spectrum expands. If the degree of crystallinity of the polymer does not exceed $70 \%$, then such a polymer can exhibit regions of maxima $\operatorname{tg} \delta(\mathrm{T})$, which are characteristic of the biocomposites studied by us. In addition, in crystallizing polymers, there are possible regions of maxima $\operatorname{tg} \delta$ of dielectric losses associated with transitions from one crystalline modification to another, local motion of macromolecules in crystalline regions, molecular motion of sections of macrochains that form folds on the surface of crystallites. Very important for determining the mechanisms of molecular motion responsible for the regions of maxima of dielectric losses are dielectric losses in the region of the maximum, which depend on the degree of crystallinity of the material. These regions of maxima of dielectric losses, the intensity of which decreases with an increase in the degree of crystallinity, are associated with molecular motion in the amorphous regions of the partially crystalline polymer.

\section{Compliance with ethical standards}

\section{Acknowledgments}

We wish to acknowledge the Head of laboratory of Department of Physics engineering and Electronica Aliyeva Sharafkhanim Vagif, for his immense assistance during the research period.

\section{Disclosure of conflict of interest}

We (Eldar Mekhraly Gojayev and Vefa Vali Salimova) the authors of the article "Dielectric properties of bionanocomposites modified by fish scales" wish to state that there are no conflicts of interests in this our research article.

\section{References}

[1] Turik AB, Rodinin MYu. Dielectric losses in materials with a limited region of the relaxation time distribution // letters to JTP. 2010; 36(1): 37-43.

[2] Berlin AA. Modern polymer composite materials. - Soros Educational Journal. 1995; 1: 57-65.

[3] Olena Yakovenko, Ludmila Matzui, Ganna Danylova, Victor Zadorozhnii, Ludmila Vovchenko, Yulia Perets, Oleksandra Lazarenko. Electrical Properties of Composite Materials with Electric Field-Assisted Alignment of Nanocarbon Fillers Nanoscale Res Lett. 2017; 12: 471.

[4] Gojayev EM, Aliyeva Sh V, Khalilova XS, Jafarova GS, Jabarov SH. The dielectric properties and electrical conductivity of LDPE modified by fillers of biological origin International Journal of Modern Physics B Vol. 33 (2019) 1950309 (10 pages) c World Scientific Publishing Company.

[5] EM Gojayev, ShV Aliyeva, VV Salimova, SH Jabarov, Yu Meshalkin. The Influence of UV Irradiation on Dielectric Properties of Biocomposites Surface Engineering and Applied Electrohemistry. 2020; 56 N6: 740-745. 